\title{
Approche clinique du diagnostic anténatal des anomalies chromosomiques
}

\author{
M Herlicoviez
}

\author{
Clinique de Gynécologie-Obstétrique, Centre Hospitalier Universitaire, Caen, France
}

(28 Réunion de la Société Française pour l'Étude de la Fertilité; Paris, 19-21 octobre 1989)

\begin{abstract}
Résumé - Face au diagnostic anténatal des anomalies chromosomiques, l'obstétricien va devoir déterminer les situations à risque où un caryotype fœtal sera réalisé. En dehors de la grossesse, les indications seront définies par un conseil génétique qui analysera des données classiques comme l'âge des géniteurs, les antécédents d'avortements à répétition, d'enfant présentant une anomalie chromosomique ou une malformation, de mort fœtale in utero... Pendant la grossesse, les signes cliniques d'alerte, en particulier hydramnios et oligoamnios mérient d'être connus, mais l'essentiel des caryotypes anormaux est détecté à partir des signes d'appel échographiques (11,3\% en moyenne). Le diagnostic de trisomie 21 fait actuellement l'objet de nombreuses études tant par la description de petits signes échographiques (brièveté du fémur; épaisseur de la nuque...) que par l'apparition de critères biologiques nouveaux : ainsi, le dosage de l'hCG dans le sang maternel permettant de définir une population à risque d'anomalie chromosomique dans laquelle on proposera un caryotype fœtal. Les modalités du prélèvement fœetal, amniocentèse précoce ou tardive, prélèvement des villosités choriales au $1^{\text {er }}$ trimestre, mais aussi éventuellement au $2^{\theta}$ voire au $3^{\theta}$ trimestre, ponction de sang fœtal, seront déterminées en fonction de la date du risque d'anomalie chromosomique, mais aussi de complications liées au prélèvement et enfin de l'expérience de chaque centre. Schématiquement, on pourrait proposer : en cas de risque élevé prévisible avant la grossesse, un prélèvement villositaire entre 9 et 11 semaines; en cas de risque faible (âge maternel par exemple) une amniocentèse ou un prélèvement villositaire précoce; en cas de risque élevé (malformation échographique), suivant la date une amniocentèse, un prélèvement villositaire tardif ou une ponction de sang fœetal.
\end{abstract}

diagnostic anténatal / caryotype fœetal / anomalies chromosomiques / homme

Summary - Clinical approach to prenatal diagnosis of chromosomal aberrations. In the case of prenatal diagnosis of chromosomal aberrations, the obstetrician has to determine the high risk situation for which a fetal karyotype examination has to be done. Before pregnancy, the karyotyping indications are defined by genetic consultation in order to analyze classical data such as the parents' age, repeated spontaneous abortions, previous child with a genetic defect, fetal malformations or intrauterine death... During pregnancy, clinical warning signs, in particular, anomalies in the amniotic fluid and fetal growth retardation should be recognized, but most abnormal karyotypes are detected by ultrasonographic examination (average: $11.3 \%$ ). Down's syndrome diagnosis is presently evaluated by some more specific ultrasonographic signs such as fetal nape or femur length measurements and by new biological parameters such as hCG assay. The sampling method for karyotyping, early or late amniocentesis, first trimester but also second and third trimester placental biopsy or cordocentesis will be chosen in accordance with the importance of the chromosomal aberration expected, gestational age, procedure complication risks and the obstetrician's experience. The following examinations could be proposed: in high risk cases determined before pregnancy, a chorionic villus sampling should be done between the 9th and 11th weeks of gestation; in low risk cases such as advanced maternal age, a first trimester chorionic villus sampling or a second trimester amniocentesis 
could be chosen; in the case of Down's syndrome, warning signs, for example ultrasonographic or biological parameters, a second trimester placental biopsy to relieve the parents' anxiety; in high risk cases such as ultrasonographic malformations, late placental biopsy or cordocentesis.

prenatal diagnosis / chromosomal aberrations / fetal karyotype / human

\section{INTRODUCTION}

Face à la priorité qu'est le diagnostic anténatal des anomalies chromosomiques, l'obstétricien va devoir déterminer les situations à risque où un prélèvement fœtal sera pratiqué.

Seront envisagés successivement : en dehors de la grossesse ou au début de celle-ci, les données anamnestiques réunies au cours d'un conseil génétique; pendant la grossesse, les signes d'appel cliniques, échographiques et biologiques.

A partir de là, le choix de la méthode de prélèvement du caryotype fœetal est discuté en fonction du signe d'appel considéré, de l'âge de la grossesse, du risque de la méthode et de l'expérience de chaque équipe.

\section{SIGNES D'APPEL}

\section{En dehors de la grossesse}

L'établissement d'un conseil génétique permet de prévoir les situations à risque d'anomalie chromosomique; il en découlera l'indication à réaliser un caryotype fœtal au cours de la grossesse à venir.

\section{L'âge des géniteurs}

Le risque de trisomie augmente avec l'âge maternel en raison d'une fertilisation tardive de l'ovocyte. Les statistiques établies par le CEBIOP de 1980 à 1985 (Boué,
1989) montrent que le risque d'anomalie chromosomique qui se situe dans la population générale à moins de $0,5 \%$ passe à 38 ans à $1,4 \%$, dont $0,77 \%$ pour les trisomies 21 ; à 40 ans à $2,38 \%$ dont $1,32 \%$ pour les trisomies 21 ; à 42 ans à $4,25 \%$ dont $2,69 \%$ pour les trisomies 21 ; à 45 ans et plus à $9,11 \%$ dont $6,07 \%$ pour les trisomies 21.

Au total, à partir de 38 ans sur un ensemble de 26704 examens, 2,56\% d'anomalies chromosomiques dont $1,57 \%$ pour les trisomies 21.

Sont ainsi diagnostiquées : des anomalies de nombre : trisomies $21,57 \%$ des cas; trisomies 18, 16\%; trisomies $13,4 \%$; $47 \mathrm{XXY}, 6 \%$; $47 \mathrm{XXX}, 5 \%, 47 \mathrm{XYY}, 2 \% ; 45$ $X, 2 \%$; des anomalies de structure : translocation équilibrées, $7 \%$, anomalies déséquilibrées, $2 \%$.

If faut remarquer que la fréquence des anomalies chromosomiques diagnostiquées au moment de l'amniocentèse est supérieure au taux qui serait observé à la naissance en raison de la survenue de morts fœtales in utero (principalement en cas de trisomie 18, monosomie $X$ et plus rarement de trisomie 21).

L'âge paternel ne semble pas avoir d'influence.

\section{Antécédents gynécologiques et obstétricaux}

\section{Avortement à répétition}

A partir de deux ou trois fausses-couches, il est nécessaire de réaliser le caryotype des géniteurs qui permettra de mettre en évidence dans 1,4 à $3,5 \%$ des cas une 
anomalie de structure chromosomique, le plus souvent une translocation équilibrée chez l'un des deux parents.

Naissance d'un enfant présentant une trisomie 21

Le risque de récurrence est faible, de moins de $1 \%$ et le diagnostic prénatal est surtout indiqué pour des raisons psychologiques.

Naissance d'un enfant malformé ou mort fotale in utero

Une enquête génétique complète et précise doit être réalisée pour dépister les situations à risque de récurrence en particulier chromosomiques.

En l'absence d'un caryotype fotal, l'indication à la grossesse suivante d'un prélèvement pour examen chromosomique dépendra du caryotype des parents (Rodesch et al, 1986).

\section{Antécédents de thérapeutiques mutagènes}

L'utilisation de chimiothérapies associées ou non à la radiothérapie dans le cadre d'affections comme la maladie de Hodgkin a été accusée d'être à l'origine de néomutations au niveau génétique ou chromosomique. II semble que ce risque soit actuellement très discuté, en particulier lorsque le traitement est ancien; le diagnostic prénatal repose sur l'échographie morphologique, et un caryotype fœtal n'est indiqué que lorsqu'un traitement maternel ou paternel est en cours au moment où débute la grossesse (Grob-Menendez et Marmor, 1989).

\section{Cas particulier de l'X fragile}

Le retard mental avec fragilité du chromosome $X$ se rencontre chez 1 garçon sur
1500. Chez les mères vectrices, le diagnostic anténatal repose sur l'examen cytogénétique des villosités choriales.

\section{PENDANT LA GROSSESSE}

\section{Signes cliniques d'alerte}

Ils doivent d'autant plus être recherchés qu'il existe actuellement un concensus limitant le nombre des échographies pratiquées pendant la grossesse.

Les métrorragies de début de grossesse celle-ci se poursuivant, ne sont plus reconnues comme facteurs de risque d'anomalie chromosomique.

Les anomalies du volume utérin sont des signes d'alerte, à condition que l'âge gestationnel soit connu avec précision : soit une augmentation de volume en relation avec une grossesse multiple, une macrosomie ou un hydramnios; soit une diminution de volume en relation avec un oligoamnios ou un retard de croissance intra-utérin.

Ces situations doivent être analysées avec soin par une échographie morphologique effectuée par un opérateur entraîné.

\section{Anomalies échographiques}

II n'est pas dans le propos de cet exposé d'entrer dans le détail des anomalies échographiques traitées dans un autre chapitre. Dans un souci de synthèse, nous rappellerons les données fournies par l'expérience pilote de dépistage des anomalies chromosomiques sur signes d'appel rapportée par Briard (1988) sur 3117 examens.

La date à laquelle est effectué le diagnostic reste tardive : $74,3 \%$ des examens sont pratiqués à 31 semaines; $16,4 \%$ à 21 semaines. 
La fréquence des anomalies chromosomiques est globalement de $11,3 \%$, mais en fonction de la nature du signe d'appel, on note les taux d'anomalies suivants: malformation échographique, $14,5 \%$; anomalies de quantité du liquide amniotique, $3,70 \%$; retard de croissance intra-utérin, $5,6 \%$.

Et si on prend en compte les associations : retard de croissance intra-utérin + anomalie du liquide amniotique, $7,2 \%$; retard de croissance intra-utérin + malformation échographique, 28,6.

C'est souligner la nécessité d'un examen échographique approfondi qui ne s'arrête pas uniquement sur une anomalie de croissance fotale ou de liquide amniotique mais cherche à mettre en évidence une anomalie morphologique (Choiset et Girard-Orgeolet, 1989; Eydoux et al, 1989). A la suite des publications de Benaceraff et al (1987), la valeur des petits signes de dépistage de la trisomie 21 (brièveté du fémur, épaisseur de la nuque...) fait actuellement l'objet d'une étude française multicentrique (Boog et Bandaly, 1987; Boog et al, 1989).

\section{Critères biologiques}

Les trois dernières années ont été marquées par la mise en route de programmes de dépistage des anomalies chromosomiques à partir de critères biologiques. Le principe de ces méthodes est le dosage dans le sang maternel de paramètres d'origine fœto-placentaire dont les taux deviennent anormaux en cas d'anomalies chromosomiques.

Les premières études ont porté sur l' $\alpha$ fœtoprotéine (AFP) sérique déjà dosée dans le dépistage des défauts de fermeture du tube neural et dont les taux abaissés permettraient de définir une population à risque de trisomie 21 . La difficulté de cette méthode est de définir un seuil qui permette de faire le maximum de diagnostics sans augmenter de façon trop importante le nombre d'amniocentèses pour caryotype fœetal.

D'autres paramètres ont été étudiés comme l'œstriol, la $\beta$-SP 1 glycoprotéine et surtout l'hCG plasmatique qui parait être actuellement la méthode la plus performante.

L'augmentation du taux d'hCG dans le sérum maternel permet selon Bogart et al (1987) de sélectionner un groupe où se situent les deux tiers des trisomies 21. Les résultats obtenus par Boué (1989) vont également dans ce sens.

\section{MÉTHODES DE PRÉLÈVEMENT}

Le caryotype fœetal peut être effectué sur un prélèvement de liquide amniotique, de villosités choriales ou de sang fœtal.

\section{L'amniocentèse}

Elle reste la méthode la plus utilisée dans cette indication. Sa technique bien connue doit faire appel à une asepsie rigoureuse et à un écho-guidage soigneux afin d'en minimiser les risques et les échecs. Le prélèvement est habituellement réalisé de 16 à 20 semaines d'aménorrhée. L'amniocentèse a actuellement tendance à être abandonnée au delà de 25 semaines au profit du prélèvement de sang fotal en raison du long délai de culture, 3 à 4 semaines, voire plus selon les laboratoires. Le risque d'avortement spontané ou de mort in utero est compris entre 0,5 et $1 \%$.

\section{Le prélèvement de villosités choriales}

\section{Au premier trimestre}

La connaissance d'un caryotype anormal au premier trimestre a l'avantage de per- 
mettre la réalisation d'une interruption de grossesse dans des conditions médicales et psychologiques beaucoup plus simples qu'à partir du deuxième trimestre. Que le prélèvement soit fait à la pince à biopsie, par aspiration, par voie transcervicale ou par voie abdominale, il doit être réalisé à l'aide d'un écho-guidage de qualité, avec une asepsie rigoureuse et par un opérateur entraîné. Le pourcentage d'échecs de prélèvements est faible variant de 0,6 à $2,16 \%$ selon les auteurs et cela quelle que soit la technique utilisée. Le facteur limitant de cette méthode reste encore le risque d'avortement; il varie de 2 à $5,5 \%$ et ne paraît pas non plus dépendant de la technique utilisée, les prélèvements transabdominaux donnant toutefois les chiffres les plus faibles. Rappelons qu'au terme de 10 semaines, le risque d'avortement spontané après une échographie normale montrant un embryon vivant et bien sûr en dehors de tout prélèvement est de 2 à $3 \%$. Le pourcentage des complications liées au mode de prélèvement ne paraît donc pas supérieur à celui d'une amniocentèse dans des mains bien entraînées.

\section{Au deuxième et au troisième trimestres}

Le prélèvement villositaire peut être effectué au cours du deuxième et même du troisième trimestre de la grossesse (Nicolaïdes et al, 1986; Szabo et al, 1986a, b; Holzgreve et al, 1987; Saura et al, 1988, 1989; Chieri et Aldini, 1989). La technique utilisée est l'aspiration à la seringue de 20 $\mathrm{ml}$ après introduction d'une aiguille de 20 gauges (16 gauges après 20 semaines) de $9 \mathrm{~cm}$ de longueur à mi-distance entre la plaque basale et la plaque choriale. Seringue et aiguille ayant été rincées à l'héparine, il faut combiner un mouvement de rotation et de va-et-vient de l'aiguille en maintenant une aspiration continue avec la seringue. Le matériel villositaire aspiré est d'environ $5 \mathrm{mg}$ et permet habituellement d'obtenir 15 mitoses, qui sont étudiées par la méthode cytogénétique directe de Simoni et al (1983) avec obtention d'un résultat dans les $48 \mathrm{~h}$. Le taux d'échec varie selon les auteurs de 2 à $9 \%$ pour le prélèvement et de $5 \%$ pour l'interprétation, principalement au-delà de 35 semaines. La simplicité de cette méthode devrait permettre son extension dans l'étude des caryotypes fœtaux sur signes d'appel.

\section{Cordocentèse}

Le prélèvement de sang fœtal à l'aiguille sous guidage échographique peut être réalisé de la dix-septième semaine jusqu'au terme de la grossesse. Cette technique à la fois simple et délicate nécessite un échographe de bonne qualité et une bonne coordination entre l'écho-guideur et le préleveur.

La ponction est réalisée à l'aide d'une aiguille de 20 gauges de $9 \mathrm{~cm}$ de longueur refiée à une seringue héparinée. L'installation de la patiente requiert une asepsie chirurgicale : désinfection de la peau, gants et champs stériles, emballage de la sonde d'échographie. Après anesthésie locale, l'aiguille est enfoncée sous contrôle échographique continu jusqu'au site choisi sur le cordon : insertion placentaire ou abdominale ou portion libre; la traversée du placenta est nécessaire lorsque son implantation est antérieure. La pureté du prélèvement doit être vérifiée aussitôt en le comparant avec le sang de la mère (mesure du volume globulaire moyen, étude de l'antigène I,i et test de Kleihauer). Les résultats du caryotype sont obtenus dans un délai de $72 \mathrm{~h}$. Le taux d'échec du prelèvement est évalué à $3 \%$. Les risques de mort fœtale in utero ou d'avortement spontané sont estimés à environ $1 \%$; toutefois en cas de souffrance fœtale chronique, dans des indications comme le retard de croissance intra-utérin, les anomalies de liquide amniotique, l'anasarque ou les mal- 
formations fœtales, ce risque passerait à près de $10 \%$.

\section{STRATÉGIE DU DIAGNOSTIC ANTÉNATAL DES ANOMALIES CHROMOSOMIQUES}

Améliorer la rentabilité du diagnostic anténatal sans augmenter de façon trop importante le nombre d'examens effectués passe par la meilleure définition des populations à risque d'anomalie chromosomique. C'est à partir des critères anamnestiques, cliniques, échographiques et biologiques que pourra s'établir en collaboration étroite avec le cytogénéticien une stratégie de dépistage. Ainsi, pour la trisomie 21 , on pourrait proposer entre 16 et 18 semaines un ou des dosages biochimiques (hCG, AFP, œstriol...). Si la femme entre dans la population définie à risque d'anomalie chromosomique, on lui propose un caryotype fœetal. A l'opposé, même si elle a dépassé 38 ans, on pourrait s'en abstenir sous réserve d'un examen échographique strictement normal. En ce qui concerne le choix du mode de prélèvement fœetal, il dépend de l'importance du risque d'anomalie chromosomique, de la date à laquelle peut être fait l'examen, mais aussi de l'expérience propre de chaque équipe tant clinique que cytogénétique.

On pourrait proposer : 1) en cas de risque élevé prévisible avant la grossesse (anomalie de structure, $X$ fragile...) : prélèvement villositaire entre 9 et 11 semaines qui apparaît la méthode la plus adaptée par sa précocité et sa rapidité, à condition que le diagnostic cytogénétique soit réalisable; 2) en cas de risque faible (âge maternel, population à risque définie par les dosages biologiques effectués à 16 semaines) : amniocentèse à 17 semaines qui reste la méthode la plus utilisée en rai- son de sa simplicité et de la rareté de ses complications; prélèvement villositaire au 1 er trimestre pour les indications d'âge maternel, plus discuté en raison du risque d'avortement légèrement accru (intérêt certain en cas d'utérus cicatriciel); prélèvement villositaire au $2^{e}$ trimestre en cas d'anomalies des dosages biologiques; 3) en cas de risque élevé apparaissant en cours de grossesse (anomalie échographique du $2^{\theta}$ voire du $3^{\theta}$ trimestre): le prélèvement de sang fœtal est certainement la méthode la plus adaptée par sa rapidité et sa fiabilité mais sa technique délicate le fait réserver encore à un petit nombre d'opérateurs expérimentés; l'amniocentèse a l'inconvénient de donner des résultats trop tardifs et aléatoires à partir du $3^{\mathbf{e}}$ trimestre; le prélèvement villositaire tardif est une méthode qui devrait se développer, car bénéficiant d'une simplicité de réalisation et d'une rapidité de lecture.

\section{RÉFÉRENCES}

Benacerraf B, Gelman R, Frigoletto F (1987) Sonographic identification of second trimester fetuses with Down's syndrome. $N$ Engl J Med 317, 1371-1409

Bogart M, Pandian M, Jones O (1987) Abnormal maternal serum chorionic gonadotropin levels in pregnancies with fetal chromosome abnormalities. Prenatal Diagn 7, 623-630

Boog G, Bandaly F (1987) Le diagnostic échographique des malformations fœetales : avantages et inconvénients. Le point de vue du praticien. In: Apport de l'Échographie en Obstétrique. Vigot, Paris, 253-273

Boog G, Gillard P, Chabaud JJ (1989) Les nouveaux signes échographiques dans le dépistage des anomalies chromosomiques. In: Diagnostic et Prise en Charge des Affections Fcetales, Vigot, Paris, 161-189

Boué A (1989) Médecine Prénatale. Biologie Clinique du Fœtus. Médecine, Sciences Flammarion, Paris

Briard ML (1988a) Diagnostic anténatal des anomalies chromosomiques, Statistiques 1980-1987. Dépêche 13, 13-43 
Briard ML (1988b) Le caryotype fotal pour les associations malformatives. Bilan de l'expérience nationale. $7^{\theta}$ Séminaire de Diagnostic Anténatal des Malformations, novembre 1988

Chieri P, Aldini J (1989) Feasibility of placental biopsy in the second trimester for fetal diagnosis. Am J Obstet Gynecol 160, 581.584

Choiset A, Girard-Orgeolet S (1989) Caryotype sur signes d'appel : le diagnostic cytogénétique dans l'étiologie des anomalies ovulaires et fœtales. In: Diagnostic et Prise en Charge des Affections Fœtales III, Vigot, Paris, 145159

Eydoux P, Choiset A, Le Porrier N (1989) Chromosomal prenatal diagnosis: study of 936 cases of intrauterine abnormalities after ultrasound assessment. Prenatal Diagn 9, 255268

Grob-Menendez F, Marmor D (1989) Traitement anticancéreux et spermatogénèse. Journées de Périconceptologie, avril (1989)

Holzgreve N, Miny P, Basaraw S, Fuhrmann N, Beller F (1987) Safety of placental biopsy in the second and third trimesters. $N$ Engl $J$ Med 317, 1159-1160

Nicolaides K, Soothill P, Rodeck C, Warren R (1986) Why confine chorionic villus (placental) biopsy to the first trimester? Lancet i, 8480, 543-544
Rodesch F, Vamos E, Hayez F, Van Regemorter $N$ (1986) Malformations congénitales, situations à risque et signes d'appel en cours de grossesse. $31^{\theta}$ Congrès des Gynécologues et Obstétriciens de Langue Française. Tours, septembre 1986. J Gynecol Obstet Biol Reprod 15, 438-444

Saura R, Longy M, Vergnaud A, Leng JJ (1988) La placentocentèse, une nouvelle technique de diagnostic prénatal d'aberration chromosomique au cours du $3^{\theta}$ trimestre. J Gynecol Obstet Biol Reprod 17, 343-345

Saura R, Horowitz J, Longy M, Vergnaud A, Grison $O$ (1989) Diagnostic prénatal du deuxième et troisième trimestre par placentogenèse : à propos de 138 cas. Communication à la Société Nationale de Gynécologie et d'Obstétrique de France. Bordeaux, mai 1989

Simoni $H$, Brambati $B$, Danesino $C$, Rossella $F$. Terzolli G, Ferrari M, Fraccaro M (1983) Efficient direct chromosome analysis and enzyme determinations from chorionic villi samples in the first trimester of pregnancy. Hum Genet 63, 349-357

Szabo J, Gellen J, Szemere G (1986a) Why confine chorionic villus (placental) biopsy to the first trimester? Lancet $\mathrm{i}, 8488,1030$

Szabo J, Gellen J, Szemere G (1986b) Midtrimester chorionic biopsy. Case report. $\mathrm{Br} J$ Obstet Gynaecol 93, 512-513 